\title{
Modeling of inner filter effect in synchronous spectrofluorimetry by using partial least squares
}

\author{
A. Andrade-Eiroa, E. Vázquez-Blanco, P. López-Mahía, S. Muniategui-Lorenzo* \\ and D. Prada-Rodríguez.
}

\author{
Department of Analytical Chemistry. A Coruña University. A Zapateira, E 15071, A Coruña. Spain
}

\begin{abstract}
This paper deals with a very common problem on spectrofluorimetry: the inner-filter effect. This limitation has made spectrofluorimetry into a disadvantageous technique for the quantification of individual compounds in complex mixtures, however, a lot of papers have quantified compounds as fluorene in mixtures where other Polycyclic Aromatic Hydrocarbons (PAHs) absorb part of the energy emitted by fluorene without taking account this limitation. The inner-effect filter for fluorene is easily detectable in spectrofluorimetric measurements in mixtures where there are compounds, such as benzo(k)fluoranthene, indeno(1,2,3-cd)pyrene and pyrene. The application of methods, such as Multiple Linear Regression (MLR) to the quantification of fluorene in mixtures containing compounds capable to quench its signal, provides high errors in the analytical results. This point is carefully treated in our paper. The precise and accurate quantification of fluorene in presence of benzo(k)fluoranthene, indene(1,2,3-cd)pyrene and pyrene, was achieved by constant-wavelength synchronous fluorimetry in combination with Partial Least Squares (PLS) calibration.
\end{abstract}

Keywords. Partial least squares - polycyclic aromatic hydrocarbons - multiple linear regression - chemiometric.

\section{Introduction}

Fluorescence spectroscopy is a powerful tool in quantitative analysis owing to its great sensitivity. However, satisfactory analysis of a fluorescent multicomponent system without any separation or derivatization step is not possible when there are serious interferences in the excitation and emission spectra. For this reason, the development of techniques that improve the selectivity of fluorimetric methods is desirable; among these techniques, synchronous and derivative fluorescence spectrometries are the most commonly used. Conventionally, synchronous fluorescence spectroscopy involves the simultaneous scanning of the excitation and emission monochromators at a constant wavelength $(\Delta \lambda)$ or energy $(\Delta v)$ difference between them, obtaining spectra simpler, narrower and more distinctive than conventional spectra $[1,2]$. The combination of synchronous fluorimetry with derivative technique allows the determination of 11 PAHs in a mixture of 18 [3]; however, the mentioned method did not allow the quantification of fluorene, because part of the energy emitted by this PAH is absorbed by others in the working mixture.

Full-spectrum multivariate calibration methods, such as Classical Least Squares (CLS), Principal Component Regression (PCR) and Partial Least Squares (PLS) methods [4-8] offer the advantage of high speed on the determination of the components of interest in mixtures, because the separation steps in the procedure can be avoided. PLS multivariate calibration in combination with spectrofluorimetry has been applied by several authors for the simultaneous determination of compounds such as nafcillin and methicillin, triphenyltin, pyridoxal, pyridoxamine and pyridoxic acid in matrices such as sea water, or human serum [9,10,11]. Amador-Hernández et al. [12] applied different chemiometric techniques to solve PAHs in a mixture of six in aqueous micellar medium by linear variable angle synchronous fluorescence. They compared the results obtained by PLS, MLR and ANN (Artificial Neural Networks).

The aim of this paper is to show a detailed investigation about how to quantify a compound which undergoes innerfilter effect by spectrofluorimetry. Examples of this problem and possible solutions had never been found before in literature. So, the compounds, which are able to quench the fluorescent signals of fluorene in a complex mixture, were identified. The ability of PLS and Multiple Linear Regression (MLR) methods, to quantify fluorene in mixtures by using constant wavelength synchronous spectrofluorimetry, was described and evaluated. This is a very important subject due to other papers have published analysis of fluorene in presence of compounds capable of quenching its fluorescence signal without taking account this possibility [13$16]$. 


\section{Experimental}

\section{Apparatus}

All the synchronous spectra were obtained on a PerkinElmer LS-50B computer controlled spectrofluorimeter (Norwalk, CT, USA) equipped with a xenon discharge light source with pulses equivalent to $20 \mathrm{KW}$ for $8 \mu \mathrm{s}$ (microseconds), two monochromators for excitation and emission (Monk-Gillieson), and provided with PE-FLDM (Perkin Elmer Fluorescence Data Manager) fluorescence software for acquisition and processing of spectral data. Wavelength accuracy and wavelength reproducibility were 1.0 and $0.5 \mathrm{~nm}$, respectively. The excitation and emission slits can be varied from 2.5 to 15 and from 2.5 to $20 \mathrm{~nm}$, respectively. Fluorescence measurements were performed using standard quartz cells $(1 \times 1 \mathrm{~cm})$. All the UV-Visible spectra were obtained on a Perkin-Elmer Lambda 6 UV-Visible (Norwalk, CT, USA) spectrophotometer.

\section{Softwares}

All the statistical and mathematical parameters were obtained by using Statgraphics (Statistical Graphics Corporation, USA) or Matlab (Math Works Inc., USA) softwares.

\section{Reagents}

The PAHs used, Ace (Acenaphthene), Anth (Anthracene), $\mathrm{BaA}$ (Benz[a]anthracene), BaP (Benzo[a]pyrene), BbFt (Benzo[b]fluoranthene), BkFt (Benzo[k]fluoranthene), BghiP (Benzo[g,h,i]perileno), Chry (Chrysene), DahA (Dibenz[a,h]anthracene), Ft (Fluoranthene), Flu (Fluorene), Ind (Indene), IP (Indeno[1,2,3-cd]pyrene), Naph (Naphthalene), Per (Perylene), Phen (Phenanthrene), Pyr (Pyrene), Try (Triphenylene), were provided by ChemService (West Chester, PA, USA) or Supelco (Bellefonte, PA, USA). The sodium sulphate anhydrous was from Merck, (Darmstadt, Alemania). The hexane was from Romil (Cambridge, Great Britain).

Stock standard solutions of $1000 \mu \mathrm{g} / \mathrm{mL}$ in hexane were prepared for further solutions and preparation of different standard mixtures. A mixture of 18 PAHs were prepared with concentrations about $20 \mathrm{ng} / \mathrm{mL}$ for all compounds, except for $\mathrm{BkFt}$ and IP, whose concentrations were close to 10 and $200 \mathrm{ng} / \mathrm{mL}$, respectively. In the calibration set, in order to construct the PLS and MLR models, 61 mixtures of Flu, $\mathrm{BkFt}$, Pyr and IP, were prepared with concentration ranges from 0 to $125,25,80$ and $1200 \mathrm{ng} / \mathrm{ml}$ respectively; for each compound included in the mixture, we have selected three concentration levels: low (about $0.00 \mathrm{ng} / \mathrm{mL}$ ), mean (about the mean concentration in the range selected for each compound) and high (the highest value in the range selected for each compound). In order to study the predictive ability of the calibration models proposed, a set of 8 synthetic mixtures was prepared in the same concentration ranges that used in the calibration step.

\section{Real Samples}

The river water samples were collected from the Cundíns River (a rural river in A Coruña, NW of Spain). Both, the tap and river water samples were collected in glass bottles previously rinsed with hexane. The samples were stored at $4{ }^{\circ} \mathrm{C}$ until analyzed within $24 \mathrm{~h}$ and filtered through a NagelMachery MN GF-6 filter (Germany) before their liquidliquid extraction.

\section{Method development}

Selection of the experimental conditions is very important for achieving the accurate and precise quantification of fluorene in the presence of other PAHs.

\section{Solvent}

The solvent is one of the factors which most influences the fluorescence signal. Hexane was chosen for its good extractive percentages and because it does not show interferences with fluorescence signals [3].

\section{Wavelength intervals}

This selection was made empirically as follows: 40 synchronous spectra were recorded at $\Delta \lambda$ between 10 and $205 \mathrm{~nm}$, under the experimental conditions mentioned in this paper. The increment between intervals was $5 \mathrm{~nm}$. The interval that provided the maximum fluorescence signal for Flu was $10 \mathrm{~nm}$. Certainly, the fluorescence intensity is at a maximum when the excitation wavelength corresponds with the maximum of the excitation spectrum and when the emission wavelength corresponds with the maximum of the emission spectrum. This occurs at a $\Delta \lambda=10 \mathrm{~nm}$ for fluorene [3].

\section{Slit-widths}

The most intense and resolved peaks were obtained for values of excitation and emission slit-widths set to 2.5 and $5.0 \mathrm{~nm}$, respectively.

\section{Scan speed}

To assure the best signal to noise performance it is necessary to select a slow scanning speed. The synchronous fluorescence spectra of hexane solutions were recorded at a rate of $240 \mathrm{~nm} / \mathrm{min}$. We have proved that rates proximal to $240 \mathrm{~nm} / \mathrm{min}$ are rather enough to assure a good signal to noise performance without wasting time unnecessarily.

\section{Spectral ranges}

In general, the PLS regression method is designed to use the full spectrum for calibration. However, there are some reasons for avoiding this. Including the spectral regions containing information not related to the analytes contribute to an increase in the memory and time required to perform the statistical treatment. Consequently the selection of a number of factors could lead to some overfitting and even to larger prediction errors [8]. Therefore, the constant-wavelength synchronous spectra for Flu, in presence of the other three 


\section{Original articles}

compounds which can absorb partially the energy emitted for it at a $\Delta \lambda=10 \mathrm{~nm}$, were recorded between 280 and $315 \mathrm{~nm}$ (the spectral regions which provides relevant spectral information). For UV-Visible spectra the range was from 250 to $350 \mathrm{~nm}$.

\section{Smoothing filter}

Two types of smoothing filters, Savitzsky-Golay and Binomial were available for use. The type of filtering selected depended on which aspects of the spectra were being analyzed. We have used the Binomial filtering because it offers the advantage of a good noise reduction with minimum peak distortion.

\section{Data treatment}

The original synchronous spectra consisted of 71 fluorescence intensity $\left(\mathrm{F}_{\mathrm{i}}\right)$ values (between 280 and $315 \mathrm{~nm}$ ) for each calibration standard. PLS calibration was applied to the spectra, using the PLS toolbox for Matlab. MLR calibration method was also applied by using Matlab software. Spectral data were converted to ASCII files with the software of the spectrofluorimeter, and subsequently all data processed was performed in the Matlab operating environment. Experimental data were subjected to a mathematical treatment prior to calibration, which consisted on the process of autoscaling of all variables in relation to the inverse of the standard deviation.

PLS and MLR were applied to the quantification of fluorene in presence of BkFt, IP y Pyr which do not show fluorescence signals in the spectral region where Flu emits; however they presents high absorbance about $310 \mathrm{~nm}$ (Fig. 2). Application of PLS involves using principal component analysis (PCA) methodology to draw analytically relevant information from the calibration data matrix. For this purpose, the data matrix $A(m, n)$ obtained by recording the fluorescence of $m$ mixtures of known composition at $n$ different wavelengths is reduced as follows:

$$
\mathrm{A}=\mathrm{TB}+\mathrm{E}
$$

where: $\mathrm{T}(\mathrm{m}, \mathrm{h})$ is the score matrix; $\mathrm{B}(\mathrm{h}, \mathrm{n})$ is the loading matrix and $E(m, n)$ is the residual matrix, $h$, being the number of principal components $[\mathrm{h} \leq \min (\mathrm{m}, \mathrm{n})]$. The following set of equations are then solved by least squares:

$$
\mathrm{C}=\mathrm{T} \mathbf{v}
$$

where: $\mathbf{v}$ is the hxl vector of coefficients relating the scores to the analyte concentrations, $\mathrm{c}$, in each one of the standard mixtures solutions. During calibration, the squares solution for $\mathbf{v}$ has the form:

$$
\mathbf{v}=\left(T^{t} T\right)^{-1} T^{t} c
$$

During the prediction, the solution for the analyte concentration in the unknown sample is quantified by determining its scores, $\mathrm{t}_{x}$, from its spectrum, $\mathrm{a}_{x}$, and using the loading matrix obtained from the calibration samples [17]:

$$
\begin{gathered}
\mathrm{a}_{x}=\mathrm{t}_{x} \mathrm{~B} \\
\mathrm{a}_{x} \mathrm{~B}^{\mathrm{t}}=\mathrm{t}_{x} \mathrm{BB}^{\mathrm{t}}=\mathrm{t}_{x}
\end{gathered}
$$

From the scores and vector $\mathbf{v}$, the different mixture components are subsequently quantified by using the following equation:

$$
\mathrm{c}_{x}=\mathrm{t}_{x} \mathbf{v}
$$

On the other hand, MLR assumes that the best approach to estimating $\mathrm{C}$ from $\mathrm{A}$ is to find the linear combination of the variables in $\mathrm{A}$ that minimizes the errors in reproducing $\mathrm{C}$. It proposes a relation-ship between $\mathrm{C}$ and $\mathrm{A}$ such that:

$$
\mathrm{C}=\mathrm{AS}+\mathrm{E}
$$

where $S$ is a matrix of regression coefficients and $E$ is a matrix of errors associated with the MLR model. S is estimated by linear regression [18].

A critical step in PLS calibration is the evaluation of the number of factors to be included in the calibration model. Cross-validation in the leave-one-out mode, that is, each standard is left out of the calibration set and a model is built with the other standards. This restricted model is then used to predict the concentration of the analyte in the standard that has been left out. The program calculates the PRESS function (Predictive Residual Error Sum of Squares) for each standard and the cumulative PRESS for all the tests (CUMPRESS function) [10]. Then, the criterium of minimum in the CUMPRESS function can be followed for selecting the best number of factors.

Remember that PRESS can be defined by the following mathematical expression:

$$
\operatorname{PRESS}=\sum_{\mathrm{i}=1}^{m}\left(\mathrm{c}_{\mathrm{ie}}-\mathrm{c}_{\mathrm{i}}\right)^{2}
$$

where: $m$ is the total number of calibration samples, $c_{\mathrm{ie}}$ represents the estimated concentration, and $c_{i}$, the reference concentration for the ith sample left out of the calibration during cross-validation [18].

Other statistical parameters were also estimated. We include their mathematical definitions.

RMSD (Root Mean Squared Deviation), SEP (Standard Error of Prediction ), REP(\%) (Relative Error of Prediction) and RE (\%) (Relative Error) are defined as follow:

$$
\operatorname{RMSD}=\sqrt{\frac{\sum\left(\mathrm{c}-\mathrm{c}_{\mathrm{e}}\right)^{2}}{m}}
$$




$$
\begin{gathered}
\mathrm{SEP}=\sqrt{\frac{\sum\left(\mathrm{c}-\mathrm{c}_{\mathrm{e}}\right)^{2}}{(m-1)}} \\
\operatorname{REP}(\%)=\frac{100}{\mathrm{C}} \sqrt{\frac{\sum\left(\mathrm{c}-\mathrm{c}_{\mathrm{e}}\right)^{2}}{m}} \\
\operatorname{RE}(\%)=\frac{\left|\mathrm{c}-\mathrm{c}_{\mathrm{e}}\right|}{\mathrm{c}} \times 100
\end{gathered}
$$

where: $c$ is the real concentration, $c_{\mathrm{e}}$ the estimated concentration of one of the analytes in a serie of samples (calibration or validation) and $\mathrm{C}$ is the average real concentration of the analyte of the former series and $\mathrm{m}$ corresponds to the number of evaluated samples.

\section{PAHs extraction in tap and river water}

Samples of $500 \mathrm{~mL}$ of unspiked and spiked tap and river waters containing different concentrations of the PAHs studied, (Flu, BkFt, IP, Pyr) were liquid-liquid extracted within 3 portions of hexane $(2 \times 10$ and $1 \times 5 \mathrm{~mL})$ in a separation funnel. The hexane extracts were filtered through sodium sulphate and further measured by spectrofluorimetry in the experimental conditions described.

\section{Results and discussion}

Figure 1 shows the constant-wavelength synchronous spectra of Flu and the mixture of 18 PAHs obtained at the experimental conditions mentioned. At the interval selected $(\Delta \lambda=10 \mathrm{~nm})$ the fluorescence intensity for Flu takes its maximum value, no other compound of the mixture shows signals in this interval at the same spectral region, although some of them absorb energy emitted by it, since the signal of Flu is slightly less in the mixture than for its individual solution as result of the inner-filter effect. This effect was easily noted in complex mixtures unless the concentrations of the compounds capable of quenching the signals of Flu are extremely low.

To identify all the compounds which absorb energy emitted by Flu in the spectral range where this compound presents maximum fluorescence intensity (about $300 \mathrm{~nm}$ for $\Delta \lambda=10 \mathrm{~nm}$ ), the UV-Visible spectra of all the compounds included in the mixture of 18 PAHs were recorded (Fig. 2). The compounds capable to quench the fluorescence signal of Flu in the working mixture were BkFt and IP, and Pyr due to these compounds absorb part of the energy emitted by Flu at $310 \mathrm{~nm}$ (the spectral region where Flu emits at a $\Delta \lambda=10 \mathrm{~nm}$ ); and for these reason, have been chosen in this study.

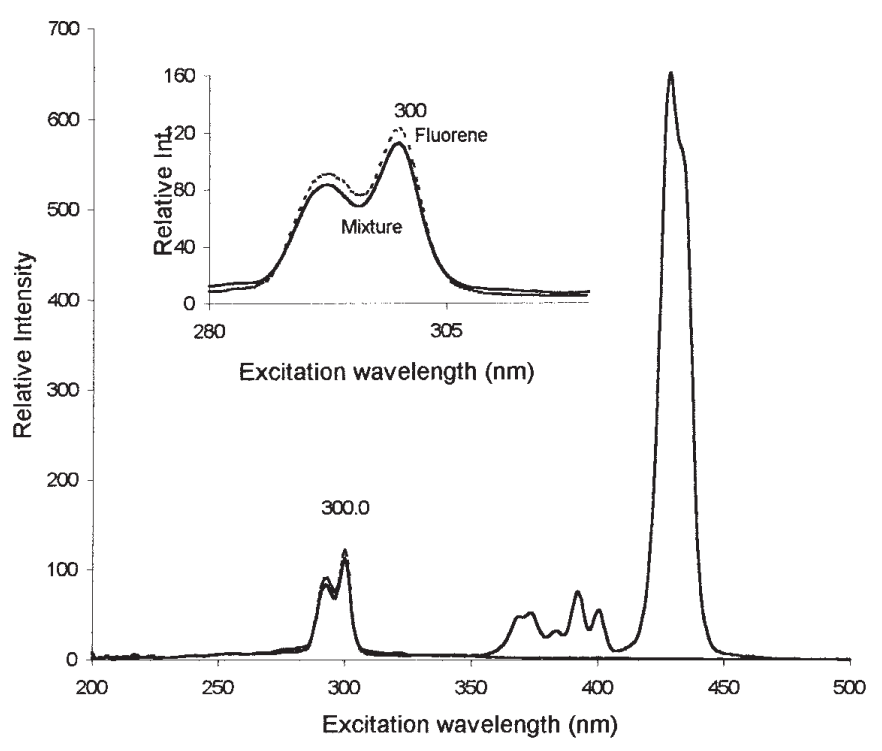

Figure 1. Constant-wavelength synchronous spectra of fluorene (dashed line) and of the mixture of 18 PAHs (continue line) at a $\Delta \lambda=10 \mathrm{~nm}$. The concentrations for all the compounds were about $20 \mathrm{ng} / \mathrm{mL}$ in all the cases except for BkFt and IP, whose concentrations were 10 and $200 \mathrm{ng} / \mathrm{mL}$, respectively.

In reference to PLS, the minimum cumulative PRESS (Fig. 3) was obtained for 1 latent variable, however, the best predictions (specially at low concentrations, $\leq 7 \mathrm{ng} / \mathrm{mL}$ ) were obtained for a number of latent variable of 3 (see table I) and, as we can see in table II, the best statistical parameters (SEP, RMSD and REP) were obtained for 2 latent variables. Therefore, selecting the minimum cumulative Press does not seem the best criterium because having a small value for CUMPRESS does not guarantee a good predictive ability, specially at low concentration levels. This is owing to a very long linear range $(2.20-125 \mathrm{ng} / \mathrm{mL})$ has been used, and the program will look for the shortest CUMPRESS, providing the more accurate results for the high values, and less accurate results for low concentrations. In summary, the number of latent variables selected was 3 .

On the other hand, a comparison between MLR and PLS is also included in table I. As we can see, the best predictions and (RE \%) were obtained by the PLS model. The predictions and RE (\%) for MLR were not acceptable. In this case MLR is not useful, due to relationship between the concentration of the compounds and the analytical signal can not be supposed completely linear and we do not know the number and the type of interactions among the analytes considered in the mixtures.

For estimating the precision of the models proposed, three spectra of three different synthetic mixtures at the same concentration were used for each concentration level included in table I. The relative standard deviations obtained for Flu ranged from $2 \%$ (for concentrations about $100 \mathrm{ng} / \mathrm{mL}$ ) to 


\section{Original articles}
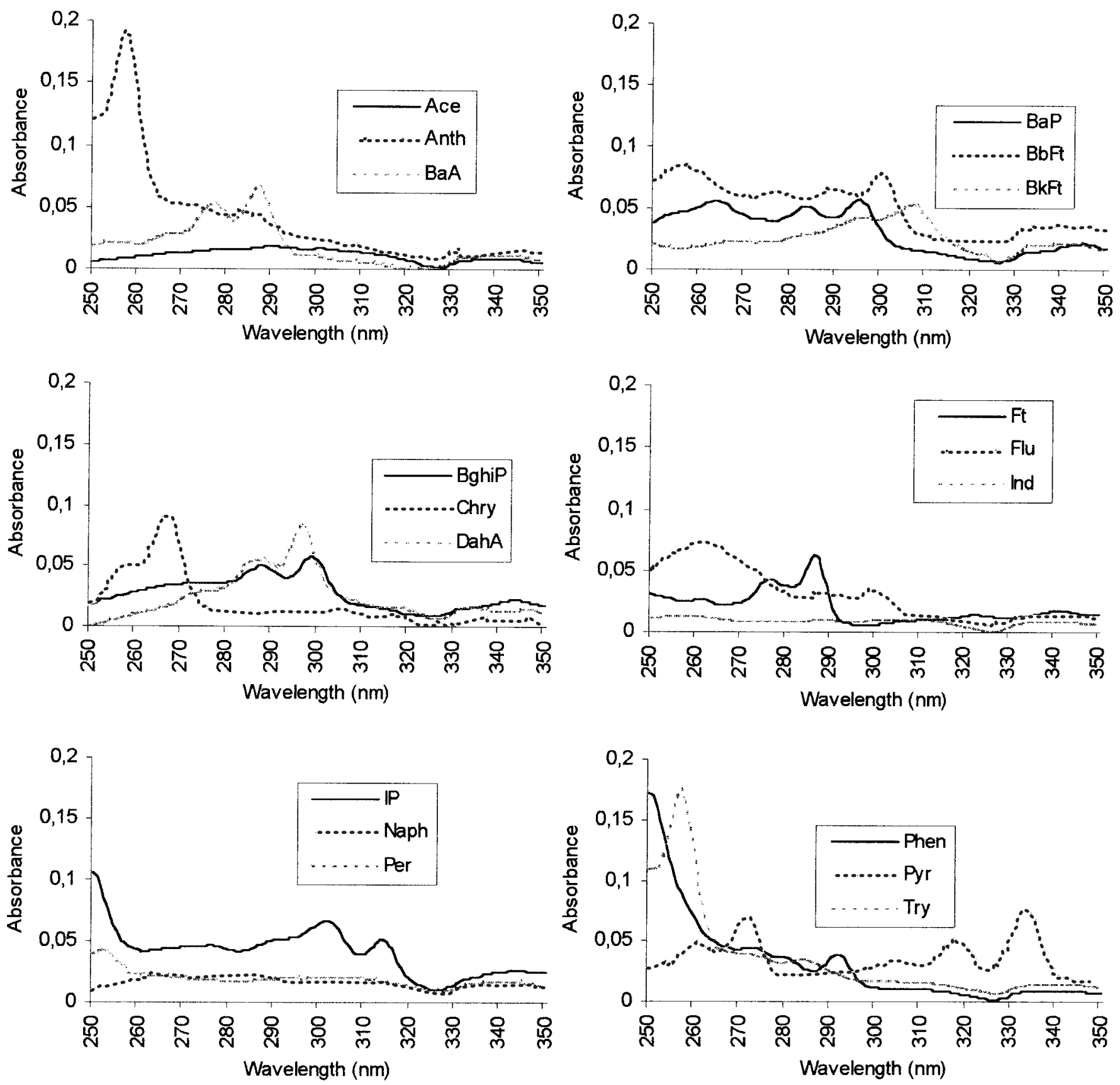

Figure 2. UV-Visible absorption spectra of the 18 PAHs studied.

$3 \%$ (for concentrations about $4 \mathrm{ng} / \mathrm{mL}$ ). So, the precision achieved was very satisfactory even at low concentrations, which allows to conclude that the models proposed are very accurate and very precise for the quantification of Flu under the experimental conditions of this paper.

Table III shows the values of the loadings for the PLS model finally selected (constructed with 3 latent variables). Each value in this table indicates the loadings for each latent variable included in the PLS model. Note that for the first latent variable, the compounds with the greatest importance were Flu and IP (they have the greatest loadings for this latent variable). The other latent variables gave more importance to compounds such as BkFt (second latent variable) or Pyr (third latent variable).

The limit of detection (LOD) of analytical methods involving a multivariate procedure, such as PLS, cannot be 
Table I. Predictions of MLR and PLS models. The RE (Relative Error), expressed in percentage, is indicated in parenthesis.

\begin{tabular}{|c|c|c|c|c|c|c|}
\hline \multicolumn{2}{|c|}{$C_{\text {real }}^{\mathrm{a}}(n g / m L)$} & $M L R$ & $P L S^{1}$ & \multicolumn{3}{|c|}{$C_{\text {found }} \mathrm{b}$ fluorene $(\mathrm{ng} / \mathrm{mL})$} \\
\hline 50.0 & $0.00,462,80.0$ & 84.8 (69.6) & $47.4(5.20)$ & $50.8(1.60)$ & $50.9(1.80)$ & $51.2(2.40)$ \\
\hline 50.0 & $26.0,462,0.00$ & 103 (106) & $48.9(2.20)$ & $52.7(5.40)$ & $53.3(6.60)$ & $53.8(7.60)$ \\
\hline 100 & $0.00,462,40.0$ & 104 (104) & $96.9(4.60)$ & $106(6.30)$ & $108(8.30)$ & $109(9.50)$ \\
\hline 50.0 & $13.0,925,0.00$ & $17.8(64.4)$ & $47.8(4.40)$ & $49.7(0.60)$ & $48.0(4.00)$ & $47.9(4.20)$ \\
\hline 100 & $13.0,462,80.0$ & 214 (114) & $98.8(1.20)$ & $104(4.20)$ & $103(3.30)$ & $102(2.80)$ \\
\hline 50.0 & $26.0,462,40.0$ & $91.7(83.4)$ & $51.5(3.00)$ & $53.70(7.40)$ & $54.3(8.60)$ & $55.5(11.0)$ \\
\hline 4.00 & $0.00,0.00,0.00$ & $-181(4425)$ & $29.8(645)$ & $6.10(52.5)$ & $4.80(16.7)$ & $4.20(5.00)$ \\
\hline 7.00 & $5.20,0.00,40.0$ & $-6.10(187)$ & $7.90(12.9)$ & $5.40(22.8)$ & $6.20(11.4)$ & $4.10(41.4)$ \\
\hline
\end{tabular}

$1,2,3,4$ number of latent variables in each case.

${ }^{\mathrm{a}, \mathrm{b}}$ Real and found concentration, respectively.

Table II. Statistical parameters (SEP, RMSD and REP) for different PLS models constructed with 1, 2 or 3 latent variables (LV). SEP.-Standard Error of Prediction, RMSD.-Root Mean Squared Deviation, REP.-Relative Error of Prediction.

\begin{tabular}{llcl}
\hline$L V$ & $S E P$ & $R M S D$ & $R E P$ \\
\hline 1 & 9.90 & 9.30 & 18.1 \\
2 & 3.70 & 3.50 & 6.70 \\
3 & 4.10 & 3.80 & 5.00 \\
4 & 5.10 & 4.80 & 9.30 \\
\hline
\end{tabular}

evaluated directly from the univariate definition of this parameter. Several approaches have been proposed to evaluate the LOD in a multivariate procedure [18-21]. In this study, this parameter was calculated from the following equation [21].

$$
\begin{gathered}
\text { LOD }=3 \varepsilon_{\mathrm{d}}|| \mathrm{b} \| \\
\varepsilon_{\mathrm{d}}=\sqrt{\frac{\sum_{i=1}^{\mathrm{NC}}\left(\mathrm{d}_{i}^{\text {obs }}-\mathrm{d}_{i}^{\text {calc }}\right)^{2}}{\mathrm{NC}-1}}
\end{gathered}
$$

where:

$\|\mathrm{b}\|$ is the square root of the sum-of-squares of each element in vector $\mathbf{b}, \mathbf{b}$ being the regression vector obtained in the PLS calibration step.

$\varepsilon_{\mathrm{d}}$, is indicative of the random instrumental error in the response spectrum.

$\mathrm{d}^{\text {obs }}$ is an experimental response vector for a sample.

$\mathrm{d}^{\mathrm{cal}}$ is its response vector calculated from the score and loading matrices obtained in the decomposition of the response matrix performed by the PLS procedure in the calibration step.

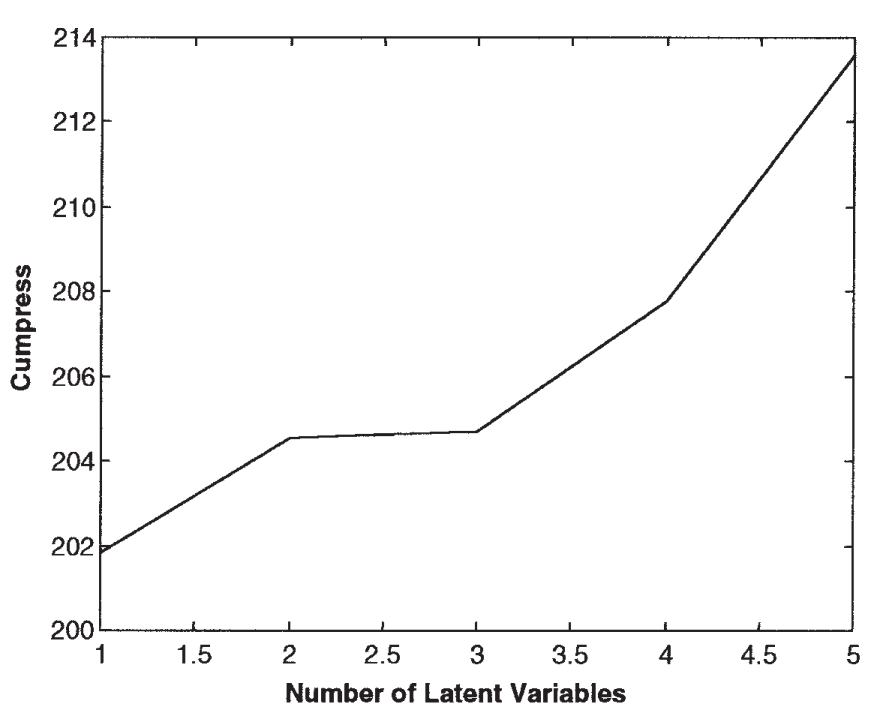

Figure 3. Minimum Cumulative Press versus number of latent variables.

$\mathrm{NC}$ is the number of $\mathrm{F}_{\mathrm{i}}$ (Fluorescence Intensity) values for each sample.

The LOD was evaluated from four different synthetic mixtures whose concentrations in $\mathrm{ng} / \mathrm{mL}$ for Flu, BkFt, IP and Pyr, respectively were the following: 0.00, 13.0, 80.0, $462 ; 2.00,13.0,925,80.0 ; 2.00,26.0,462,80.0 ; 2.00,26.0$, $0.00,80.0)$. The LOD obtained for Flu, expressed as the mean value, was $0.50 \mathrm{ng} / \mathrm{mL}$ with a standard deviation of 0.10 which is of the same order of magnitude as such as estimated according to Miller and Miller [22] for individual solutions of Flu $(0.2 \mathrm{ng} / \mathrm{mL})$.

In reference to linear ranges, considering that the innerfilter effect offers only a little deviation of linearity (because the quenching of the Flu is never greater than $15 \%$ [3]), it can be concluded that the linear range for Flu in mixtures is near to its linear range in individual solutions, 2.20 to $125 \mathrm{ng} / \mathrm{mL}$. 


\section{Original articles}

Table III. Values of the loadings of each latent variable (LV) for the PLS model selected.

\begin{tabular}{lccc}
\hline COMPOUND & LV1 & LV2 & LV3 \\
\hline Flu & 0.9633 & -0.2838 & 0.0710 \\
Pyr & -0.1586 & -0.4569 & 0.7957 \\
BkF & 0.0807 & 0.7440 & -0.2377 \\
IP & 0.2007 & 0.3963 & 0.5526 \\
\hline
\end{tabular}

Table IV. Simultaneous PLS-synchronous spectrofluorimetry determination of fluorene in spiked samples of tap and river water.

\begin{tabular}{lccc}
\hline & \multicolumn{2}{c}{$C_{\text {added }}^{*}(\mathrm{ng} / \mathrm{mL})$} & \multicolumn{2}{c}{$C_{\text {found }}$ fluorene $(\mathrm{ng} / \mathrm{mL})$} \\
Flu & $B k F t$, IP, Pyr & Tap water & River water \\
\hline 0.00 & $0.00,0.00,0.00$ & $\mathrm{ND}$ & $\mathrm{ND}$ \\
50.0 & $0.00,462,80.0$ & 49.7 & 47.2 \\
50.0 & $26.0,462,0.00$ & 50.1 & 46.8 \\
100 & $0.00,462,40.0$ & 98.5 & 90.3 \\
50.0 & $13.0,925,0.00$ & 52.1 & 45.3 \\
100 & $13.0,462,80.0$ & 99.4 & 88.6 \\
50.0 & $26.0,462,40.0$ & 51.3 & 46.5 \\
4.00 & $0.00,0.00,0.00$ & 3.9 & 3.5 \\
7.00 & $5.20,0.00,40.0$ & 6.7 & 6.1 \\
\hline
\end{tabular}

* The concentrations are to hexane extracts referred.

The PLS model selected was applied to synchronous spectra data of hexane extracts (obtained by liquid-liquid extraction) of tap and river waters spiked samples (analyses were done in duplicate in each case). The results found are given in table IV and, as we can see, they were satisfactory in all cases. In summary, PLS appears suitable for the analysis of real water samples.

\section{Conclusions}

This paper deals with a very common limitation in spectrofluorimetry: the inner-filter effect. This limitation was often ignored in previous papers and lead to errors in the analytical results if a incorrect model is applied for its quantification. The fluorescence signal of Flu in a complex mixtures of 18 PAHs, is significantly less compared to its signal in individual solutions. This is owing to that certain PAHs absorb partially the energy emitted by Flu at a $\Delta \lambda=10 \mathrm{~nm}$ and $310 \mathrm{~nm}$. The identification of these PAHs (BkFt, IP and Py) was achieved by examining their UV-Visible spectra.

The PLS and MLR algorithms were applied to construct a model capable to predict Flu in mixtures including the analyte of interest and the compounds capable to quench its fluorescence signal. The MLR has provided unsatisfactory results, while the PLS has demonstrated to be able to take into account the inner filter effect and predict the concentration of Flu in an accurate and precise way.

Moreover, the PLS model selected (constructed with tree latent variable) in combination with constant-wavelength synchronous fluorimetry, was suitable for the determination of fluorene (in presence of the other PAHs involved in the inner-filter effect) in spiked real water samples (tap and river waters).

\section{Acknowledgements}

We gratefully aknowledge the Consellería de Presidencia de la Xunta de Galicia for the concession of bursaries to Áurea Andrade Eiroa.

\section{References}

1. Lloyd, J. B.F. Nature 1971, 231, 64-65.

2. Inman, E.L. Jr.; Winefordner, J.D. Anal. Chem. 1982, 54, 2018-2022.

3. Andrade Eiroa, A.; Vázquez Blanco, E.; López Mahía, P.; Muniategui Lorenzo, S.; Prada Rodríguez, D. Analyst 1998, 123, 2113-2117.

4. Wold, H. Research Papers in Statistics; David, F., Ed.; Wiley: New York, 1966; pp 411-444

5. Wold, S.; Martens, H.; Wold, H. Multivariate Calibration Problem in Chemistry Solved by PLS; Ruhe, A., and Kagstrom, B., Ed.; Springer; Heidelberg, 1983; pp 286-293.

6. Haaland, D.M.; Thomas, E.V. Anal. Chem. 1988, 60, 11931202.

7. Thomas, E.V.; Haaland, D.M. Anal. Chem. 1990, 62, 10911099.

8. Martens, H.; Naes, T. Multivariate Calibration; Wiley: New York, 1989.

9. Murillo, J.A.; Alañón, A; Fernández, P.; Muñoz de la Peña, A.; Espinosa-Mansilla, A. Analyst 1998, 123, 1073-1077.

10. Leal, C.; Granados, M.; Beltrán, J.L.; Compañó, R.; Prat, M.D. Analyst 1997, 122, 1293-1298.

11. Berzas Nevado, J.J.; Murillo Pulgarín, J.A.; AmadorHernández, J.; Gómez Laguna, M.A. Analyst 1998, 123, 483488.

12. Amador-Henández, J.; Cladera, A.; Estela, J.M.; López-deAlba, P. L.; Cerdá, V. Analyst 1998, 123, 2235-2242.

13. Rubio Barroso, S.; Kayali, M.N.; Polo-Díez, L.M. Química Analítica, 1993, 12, 187-191.

14. Rubio Barroso, S.; López López, D.; Val Ontillera, C.; Polo Díez, L. Química Analítica, 1991, 10, 127-136.

15. Files, L. A. Jones, B. T. Hanamura, S. and Winefordner, J. D. Anal. Chem. 1986, 58, 1440-1443.

16. Lorber, A. Anal. Chem. 1986, 58, 1167-1172.

17. Múñoz de la Peña, A.; Durán-Merás, I.; Moreno, M.D.; Salinas, F.; Martínez Galera, M. Fresenius J. Anal. Chem. 1995, 351, 571-576.

18. Beebe, K.R.; Kowalski, B.R. Anal. Chem. 1987, 59, 1007A$1017 \mathrm{~A}$

19. Clayton, C.A.; Himes, J. W.; Elkins, P. D. Anal. Chem. 1987, 59, 2506-2514.

20. Bauer, G.; Wegscheider, W.; Ortner, H.M. Fresenius J. Anal. Chem. 1991, 340, 135-139.

21. Booksh, K.S.; Kowalski, B.R. Anal. Chem. 1994, 66, 782791A.

22. Miller, J.C.; Miller, J.N. Statistics for Analytical Chemistry; Ellis Horwood: Chichester, 1988, pp 103-119. 